\title{
A Haptic Gaming System for Tactile Textures and 3D Shapes Discrimination
}

\author{
Sung Min Kim and Mee Young Sung \\ Department of Computer Science and Engineering \\ Incheon National University, Incheon, Korea \\ ksmvision@incheon.ac.kr,mysung@incheon.ac.kr
}

\begin{abstract}
Haptics-enabled virtual reality technologies allow usersto sense forke feedback and tactile feedback while manipulating 3D objects. This paper proposes d haptic ganing system which can contribute to improve the ability for discerning tactlle textures and $3 D$ shapes by using force and tactile feedbacks of the users' fingers. Our haptic gaming system consists of three games: "Haptic Puzzle" for tactile texture cognition, "Shape Puzzle" for 3D geometric object cognition, and "Position Puzzle", for 3 D space cognition. Through a number of experiments, we have confirmed the effectiveness of our yystem on enhancing the tactile textures and 3D shapes recognition. The discrimination test of four haptic properties (stiffness, damping, static friction, and dynamic friction) led us to confirm that the users were least sensitive with damping property, and the damping changes were also highly confused with stiffness changes. We also found that the stiffness and damping properties are more influential than friction preperties for discriminating the objects via the tactile texture recognition test. The final experiment demonstrated that the visuo-haptic integrated recognition was more adyantageous than single haptic recognition. Moreover, this work showed the feasibility that the proposed system can be used for evaluating one's haptic visual discrimination capability.
\end{abstract}

Keywords: haptics, virtwalreality, gaming, discrimination, tactile textures, $3 D$ shapes

\section{Introduction}

Virtual reality (VR) provides an illusion of the reality. Virtual reality technologies are popularly used/in computing systems in the fields of education, entertainment, training, medicine, efo. [1-2]. By definition, virtual reality is a computer mediated environment that can simulate physical presence in places in the real world or imagined worlds. Virtual reality environments can be realized by a careful implementation of five human sensory phenomena: the visual perception, auditory perception, haptic perception, olfactory (smell) perception, and palatal (taste) perception.

Among these, haptic perception is the most prevailing sensation following after vision and audition in computer-human interactions, and haptics is the technology devised to apply the senses of touch to make the interactions more effective [3-4]. Although the haptic support can provide virtual environments with more immersive and interactive qualities, the research for this field is still in its infant stage. Recent studies are still limited in their focus on implementing a supplementary sensory channel for the blind [5-8].

Among the many possible applications of the technology, this paper will focus mainly on the haptic VR gaming because of its attractive appeal. TheraGame is an example of a VR rehabilitation game that uses a simple webcam to treat neurological impairment [9]. Another 
one is the Ubi-Pen Puzzle [10] game which makes users to match the puzzle by selecting, moving, rotating and resizing a polygon with a vibratory stylus pen, providing tactile cues when matched. Currently, VR rehabilitation researches are limited to audiovisual senses without any haptics, or just a simple force and vibratory senses. There is still no technology that incorporates both force and tactile sensations at the same time.

The objective of our study is to develop a haptic VR gaming system that can be used for helping the impaired as well as seniors and children to improve their ability to discern tactile textures and 3D shapes. Our proposal is based upon the technology used in three games: "Haptic Puzzle" for tactile texture cognition, "Shape Puzzle" for 3D geometric object cognition, and "Position Puzzle" for 3D space cognition.

Two subjective tests were performed to validate our proposal. The first was a test based on SUS (System Usability Scale) [11], and the second was a haptic reality test Additionally, a haptic properties discrimination test, tactile texture recognition test, and haptie 3D shape recognition test were undertaken to ensure the effect of our system on improving sensitive discrimination capability.

\section{Background}

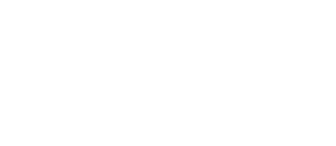

Recently, haptic VR technology is rapidly growing, and many experimental systems are being created, which are mainly based upon tactile andforce interactions [12-13]. Haptic feedback can be distinguished by the four properties: stiffness, damping, static friction, and dynamic friction. These properties are relevant to physical forces such as spring force, damping force, and friction force. 1

Spring Force: Because a spring can store mechanical energy, the spring force is commonly used for spring-damper haptic rendering. Hooke's law explains that, under the convention that $F$ is the resforing (reaction) force exerted by the spring on whatever that is pulling its free end, the following equation can be formulated: $F=-K x$ (where $F$ is the force, $K$ is the spring constant (its stiffness), and $x$ is the distance). Since the direction of the restoring force is opposite to that of the displacement, the spring force can be modeled in terms of the stiffness of an ojject. The spring constant value $k$ influences the stiffness of a spring-damper object when the distances of objects are the same.

Damping Force: Adamper is a mechanical device that resists motion via viscosity. Linear damping occurs when a potential oscillatory variable is damped by an opposing influence, which is in direct proportion to the instantaneous rate of change and the velocity or time derivative of the variable itself. In physics and engineering, damping may be mathematraky modelled as a force synchronous with the velocity of the object but opposite (n) direction. If such force is also proportional to the velocity, as for a simple mechanical viscous damper, that can be expressed as $F=-B v$ (where $F$ is the force, $B$ is the damping coefficient, and $v$ is the velocity). What it means is that, the damping force can be modeled for the viscosity of an object, and the damping coefficient value $B$ influences the viscosity of a spring-damper modeled object when the velocities of objects are identical.

Friction Force: Friction is a surface force that opposes relative motion. The frictional force is directly related to the normal force which acts to keep two solid objects separated at the point of contact. There are two broad classifications of frictional forces: static friction and dynamic (=kinetic) friction. The static friction force $\left(F_{s f}\right)$ will exactly oppose the forces applied to the object parallel to the surface of contact, up to the limit that is specified by the multiplication between the coefficient of the static friction $\left(\mu_{s f}\right)$ and the normal force 
$\left(F_{N}\right)$. The magnitude of the static friction force satisfies the inequality: $0 \leq F_{s f} \leq \mu_{s f} F_{N}$ (where $F_{s f}$ : the static friction force, $\mu_{s f}$ : the coefficient of static friction, $F_{N}$ : the normal force). The dynamic friction force $\left(F_{k f}\right)$ is independent of both the forces applied and the movement of the object. Thus, the magnitude of the force equals: $F_{k f}=\mu_{k f} F_{N}$ (where, $F_{k f}$ is the dynamic friction force, $\mu_{k f}$ is the coefficient of dynamic friction, and $F_{N}$ is the normal force). For most surface interfaces, the coefficient of dynamic friction is less than the coefficient of static friction. Consequently, the friction force can be modeled after the surface texture of the object, and the friction coefficient values $\mu_{k f}$ and $\mu_{s f}$ influence the texture when the normal forces of the objects are equal.

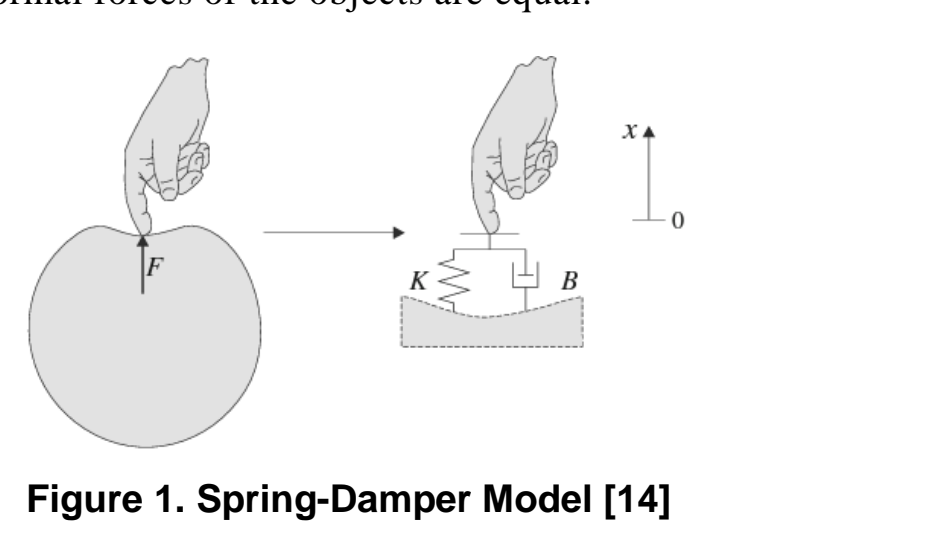

The most common way Øf modeling stift and grounded surface is based on the model consisting of a parallel connection between a spring with stiffness $K$ and a damper with viscosity $B$, as shown in Figure 1 As $x=0$ represents the undistorted state of an object while $x<0$ showing the inside of the object's boundaries, the modeled contact force on the virtual hand as shoyn in Figure 1 equals:

$$
F= \begin{cases}-(K x+B \dot{x}) & \text { for } x<0 \wedge \dot{x}<0 \\ -K x & \text { for } x<0 \wedge \dot{x} \geq 0 \\ 0 & \text { for } x \geq 0 .\end{cases}
$$

(where $F$ : the force, $K$ : the stiffness, $B$ : the viscosity, $x$ : the distance, $\dot{x}:$ the movement velocity)

In this case, the viscous damping behaves as a directed damper, which becomes active when the object is pressed and passive when the pressure is withdrawn. This enables a stable and damped contact with the object, creating a realistic contact rendering [14].

The four properties (stiffness, damping, static and dynamic friction) can produce various patterns of texture when they are synchronized with graphical properties of the objects. For example, if the four properties are respectively set to the values of 1.0, 1.0, 0.0 and 0.0 (those values are normalized to 1), such combination can generate a feeling of touching a glass object. However, since the sense of touch comes from human sensory experiments, it is difficult to define the optimal values for the four properties without actual human experiments. 


\section{The Proposed System}

Our haptic gaming system is designed to enhance the discrimination capability of tactile textures and 3D geometric shapes. In this section, we present our system which consists of following three games:

- "Haptic Puzzle" for matching the same tactile sense of the target texture

- "Shape Puzzle" for matching the shape of the target 3D figure object

- "Position Puzzle" for matching the 3D position of the target 3D coordinates

\subsection{Overview}

We briefly explain three puzzles that are developed to satisfy the objective of our stydy for discerning tactile textures and 3D shapes.

Haptic Puzzle: The game starts with the target texture on the left and andomly selected nine different tactile senses presented on the right as shown in Figure 2. Users can feel the target texture and all nine of the presented textures with haptic deviee at any time. They then go on to choose the same texture as the target texture among nine choices (meat, wood, rock, glass, leather, bone, sand, jelly, and orange).

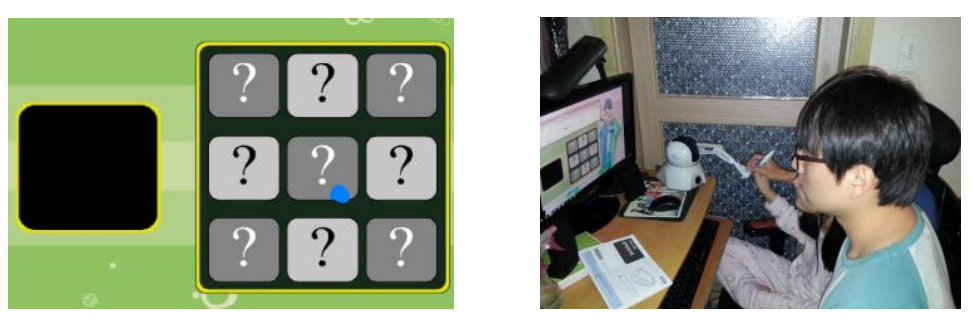

Figure 2. Screen Shots of Haptic Puzzle

Shape Puzzle: Finst, this game presents a big question mark. Then it randomly assigns behind the question mark a 3D geometric object. The possible objects are: sphere, torus, tetrahedron (composed of 4 regular triangular faces), hexahedron (6 regular square faces), octahedron (8 regular triapgle faces), dodecahedron (12 regular pentagonal faces), and icosahedron (20 regular triangle faces). Users can probe the 3D structure and figure out what it is.
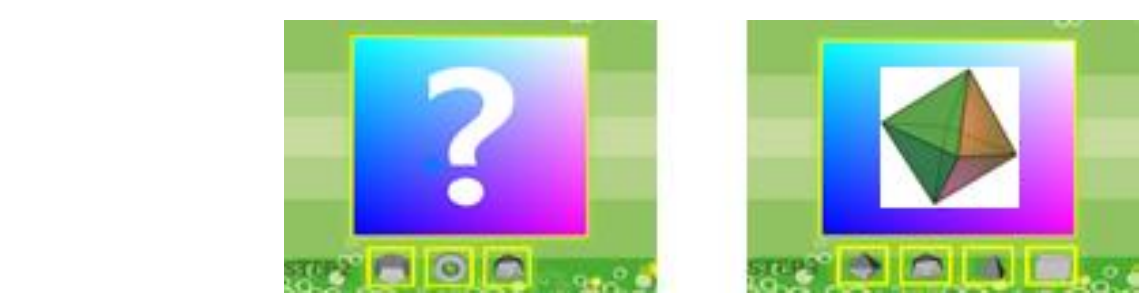

Figure 3. Screen Shots of Shape Puzzle

Position Puzzle: At the beginning, a cursor appears on the dark screen, and then it suddenly disappears to the place where an invisible wolf is placed. This game allows users to guess the position where the cursor has disappeared. Users can move the haptic device to their guessed positions and feel the tactile feedback hints of the invisible wolf. The game ends when the users find the invisible wolf and click on the button of the haptic device. If the 
answer is correct, the level of the game increases, and the size of the invisible wolf becomes smaller than that of the previous level.
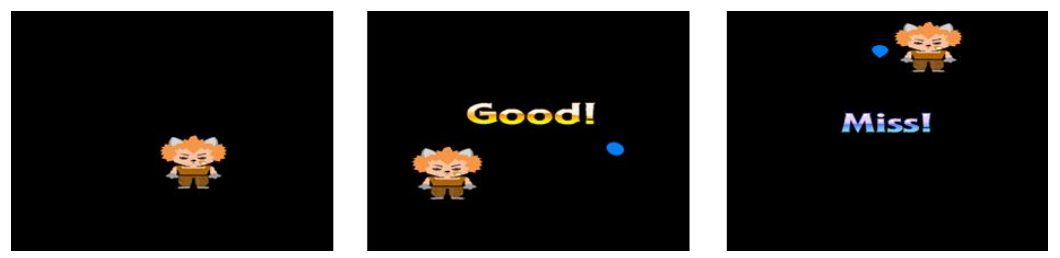

Figure 4. Screen Shots of Position Puzzle

\subsection{Design and Implementation}

The software structure of the proposed system is summarized in the Figure 5. The system is composed of four layers: Platform, Interface, Renderer, and Resoutrce. Three puzzle applications are placed above the Resource layer. This section describes the platform and our intrinsic modules developed for this system.

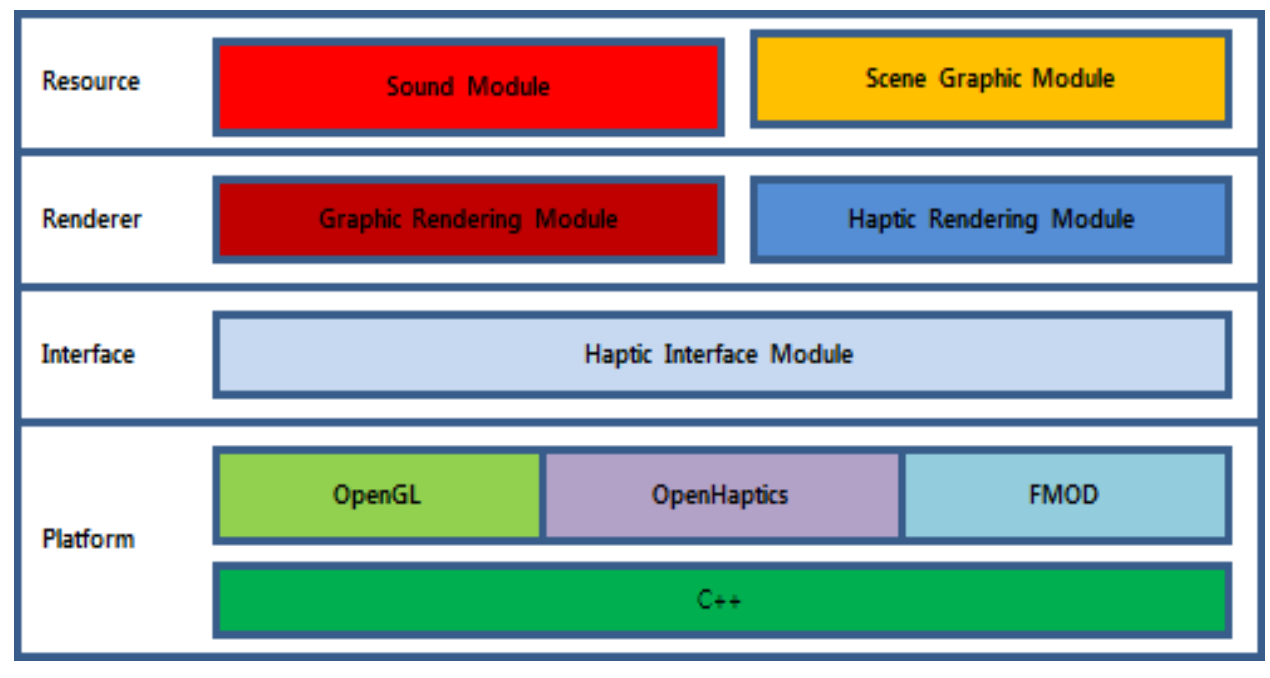

Figure 5. Software Structure of the Proposed System

Platform: The system hardware includes PHANToM Omni haptic device [15] which provides si spatial degrees of freedom (forward/back, up/down, left/right, pitch, yaw, and roll) and three force degrees of freedom (forward/back, up/down, and left/right). Haptic rendeking is implemented using SensAble OpenHaptics Toolkit. Graphic rendering is coded with OpenGL (Open Graphics Library) [16]. Sound rendering is done with FMOD [17] (a set of audio content creation tools made by Firelight Technologies that play music files of diverse formats).

Haptic Interface Module: This module communicates with the haptic device. It sends and receives the coordinates of the haptic device point and button click events. HLAPI (Haptic Library API) and HDAPI (Haptic Device API) are used for controlling the PHANToM Omni haptic device. HDAPI allows low-level access for haptic rendering, whereas HLAPI allows high-level access.

Haptic Rendering Module: This module produces various patterns of texture by combining the four properties: stiffness, damping, static friction, and dynamic friction. It uses HLAPI and generates tactile sensations of touched objects when they are synchronized with 
graphical properties of the object. The greater the dynamic friction value becomes, the coarser the texture is. The greater the stiffness value is, the harder the object becomes.

Graphic Rendering Module: Graphic rendering is performed by this module using the OpenGL (Open Graphic Library). It loads game images and presents at $30 \mathrm{~Hz}$. The graphic rendering cycle is independent from the haptic rendering cycle. It uses 3D shape libraries of OpenGL for our Shape Puzzle, which are sphere, torus, tetrahedron, hexahedron, octahedron, dodecahedron, and icosahedron.

Sound Module: This module plays MP3 files using the sound middleware FMOD API, which is a set of libraries written in $\mathrm{C}$ programming language for playing sound sources. Different sound channels and different sound sources are used for different puzzles,

Scene Graphic Module: This scene graphic module loads bitmap images and renders 2D images. It also presents the movements of game characters such as walking by using mask images and sprite processing. The bit map images of 512 by 512 pixels are used for textures, and they are mapped through OpenGL functions.

\section{Experiments}

This section describes how the experiments of our proposed system were conducted. Some subjective tests for system usability and for haptic veality of our system are examined. Before the recognition tests, a discrimination test ${ }^{f}$ or the four haptic properties was undertaken. Then we analyzed the tactile texture recogtition performance and haptic shape recognition performance using our system.

\subsection{System Usability Test}

The first subjective test was based on the SUS (System Usability Scale) [11]). It was performed to test the usability of our system. Ten investigation questions were prepared to ask the immersiveness, entertainingtess, freshness, easiness, and tactile sensations of each game. The participants of the questionnaire were 30 male and female university students. The survey result alculated by SUS has the mean of 76.5 and standard deviation of 9.24. This score is relatively higher than that of TheraGame (73.8).

Table 1 illustrates the results of the system usability test for each game which was scored in the scale of 1 to 5 for their four categories.

Table 1. System Usability Scale
\begin{tabular}{|c|c|c|c|c|}
\hline Game & immersiveness & entertainingness & freshness & easiness \\
\hline Haptic Puzzle & 4.2 & 4.5 & 4.4 & 2.1 \\
\hline Shape Puzzle & 4.7 & 3.7 & 3.6 & 2.4 \\
\hline Position Puzzle & 4.5 & 3.9 & 4.1 & 3.3 \\
\hline
\end{tabular}

The Haptic Puzzle received high scores in entertainingness and freshness. The Shape Puzzle got the highest score in immersiveness, and the Position Puzzle earned similar scores in different categories. All of three puzzles earned low marks in easiness because of the difficulties in handling an unfamiliar device and haptic cursors in 3D space.

\subsection{Haptic Reality Test}

Our second subjective test was on the haptic reality measures. The task was to rate from 1 to 5 depending on the similarity of the artificial tactile sensations to their actual textures. The 
first step for testing haptic reality was to define the textures of the objects correctly. Currently, we can distinguish more than nine different tactile senses such as meat, wood, rock, glass, leather, bone, sand, jelly, orange, etc. Table 2 illustrates the values of the four haptic properties, obtained through repetitive trials of their combinations.

Table 2. Haptic Properties of Different Materials

\begin{tabular}{|l|c|c|c|c|}
\hline Material & Stiffness & Damping & $\begin{array}{c}\text { Static } \\
\text { Friction }\end{array}$ & $\begin{array}{c}\text { Dynamic } \\
\text { Friction }\end{array}$ \\
\hline Glass & 1.0 & 1.0 & 0.0 & 0.0 \\
\hline Jelly & 0.1 & 0.0 & 1.0 & 0.2 \\
\hline Wood & 1.0 & 1.0 & 0.2 & 0.2 \\
\hline Orange & 0.5 & 0.1 & 0.9 & 0.2 \\
\hline Meat & 1.0 & 1.0 & 0.0 & 0.2 \\
\hline Bone & 1.0 & 0.7 & 0.2 & 0.7 \\
\hline Leather & 1.0 & 0.0 & 0.1 & 0.2 \\
\hline Rock & 1.0 & 1.0 & 0.7 & 0.3 \\
\hline Sand & 1.0 & 1.0 & 0.9 & 0.7 \\
\hline
\end{tabular}

The results of this haptic reality test are summarized in Figure 6 . The reality measures are:
for glass, 4.1 for jelly, 3.7 for wood, 3.1 for ofange. 2.7 for meat, 2.5 for bone, 2.1 for leather, 1.7 for rock, 1.3 for sand (in adescending onder).

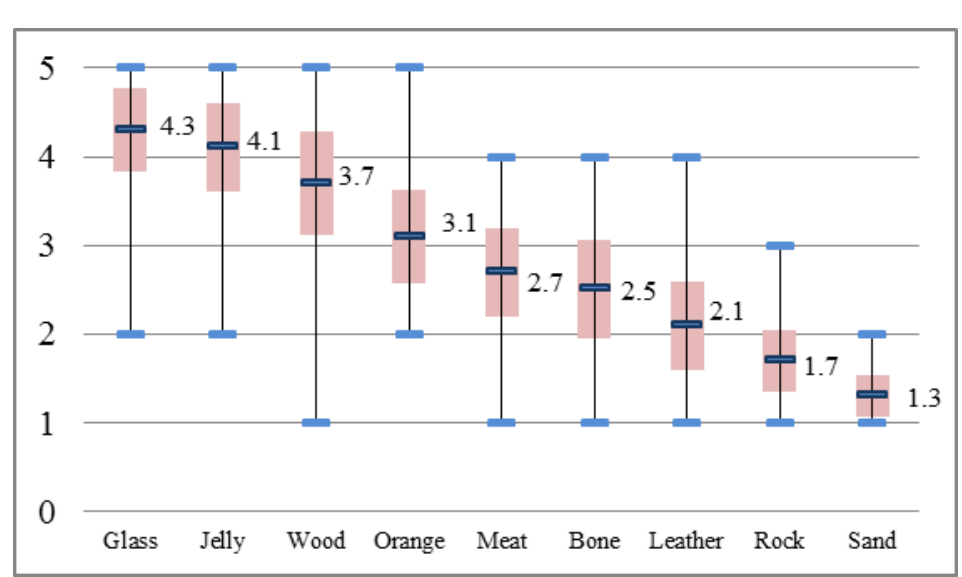

Figure 6. Result of the Haptic Reality Test

The) an ANOVA (ANalysis Of VAlue) test was performed for confirming the validity of the test results. The test was calculated from the null hypothesis $\left(H_{0}\right.$ : Each tactile reality measure has no difference) and the target samples. For its result, it was deemed statistically significant if the ANOVA test yielded a value that is not merely out of chance, assuming the truth of the null hypothesis. In other words, a statistical result is considered statistically significant, when a probability (p-value) turns out to be less than a certain threshold (significance level), often 5\% $(\alpha=0.05)$. A statistically significant result justifies the rejection of the null hypothesis, but only if the a priori probability of the null hypothesis is not high. Thus, the resulting p-value of $3.76 \times 10^{-38}$, which is less than $\alpha(0.05)$, justifies the rejection of the null hypothesis, and we concluded that each tactile reality measure has the difference. 
Table 3. ANOVA Test for the Haptic Reality Test

\begin{tabular}{|c|c|c|c|c|c|}
\hline \multirow[b]{2}{*}{ Material } & \multirow{2}{*}{$\begin{array}{c}\text { Reality } \\
(1 \sim 5)\end{array}$} & \multirow[b]{2}{*}{ Variance } & \multicolumn{3}{|c|}{ Result of ANOVA } \\
\hline & & & $\begin{array}{c}\text { Degrees of } \\
\text { Freedom }\end{array}$ & $\mathrm{F}$ ratio & P-value \\
\hline Glass & 4.3 & 0.906897 & \multirow{9}{*}{8} & \multirow{9}{*}{36.04853} & \multirow{9}{*}{$3.76 \mathrm{E}-38$} \\
\hline Jelly & 4.1 & 0.989655 & & & \\
\hline Wood & 3.7 & 1.389655 & & & \\
\hline Orange & 3.1 & 1.127586 & & & \\
\hline Meat & 2.7 & 0.975862 & & & \\
\hline Bone & 2.5 & 1.224138 & & & \\
\hline Leather & 2.1 & 0.989655 & & & \\
\hline Rock & 1.7 & 0.493103 & & & \\
\hline Sand & 1.3 & 0.217241 & & & \\
\hline
\end{tabular}

\subsection{Haptic Properties Discrimination Test}

Prior to the experiments on the recognition performances, it was interesting to examine the user sensitivity and influences of each haptic property. The stiffness defines the hardness of an object; the damping defines the viscosit of an object, the static friction; the dynamic friction defines the texture sensations.

In order to examine them, we carried out the haptic properties discrimination test to identify the differences between the users. The standard sensitivity was defined as $(0.1,0.1$, $0.1,0.1$ ) for all respective categories (stiffness, damping, static friction, dynamic friction). Then they were also redefined for each of the examinations: $(0.9,0.1,0.1,0.1)$ for the stiffness test, $(0.1,0.9,0.1,0.1)$ for the damplng test, $(0.1,0.1,0.9,0.1)$ for the static friction test, and $(0.1,0.1,0.1,0.9)$ for the dynamic friction test.

The experiments were done with the assumption that users can easily discern the obvious differences of each property. Theresults of the experiments are presented in the Table 4.

\section{Table 4. Haptic Properties Discrimination Test}

\begin{tabular}{|c|c|c|c|c|c|}
\hline & $\begin{array}{c}\text { Standard } \\
(0.1,01,0) 1,0.1)\end{array}$ & $\begin{array}{c}\text { Stiffness } \\
(0.9,0.1,0.1,0.1)\end{array}$ & $\begin{array}{c}\text { Damping } \\
(0.1,0.9,0.1,0.1)\end{array}$ & $\begin{array}{c}\text { Static Friction } \\
(0.1,0.1,0.9,0.1)\end{array}$ & $\begin{array}{c}\text { Dynamic Friction } \\
(0.1,0.1,0.1,0.9)\end{array}$ \\
\hline $\begin{array}{c}\text { Standard } \\
(0.1,0.1,0.1,0.1)\end{array}$ & 20 & 0 & 0 & 0 & 0 \\
\hline $\begin{array}{c}\text { Stiffness } \\
(0.9,0.1,0.1,0.0)\end{array}$ & 0 & 12 & 8 & 0 & 0 \\
\hline $\begin{array}{c}\text { Damping } \\
(0.1,0.9,01,0.1)\end{array}$ & 0 & 10 & 10 & 0 & 0 \\
\hline $\begin{array}{c}\text { Static F)iction } \\
(0.1,0.1,0.9,0.1)\end{array}$ & 0 & 0 & 0 & 20 & 20 \\
\hline $\begin{array}{c}\text { Dynamic Friction } \\
(0.1,0.1,0.1,0.9)\end{array}$ & 0 & 0 & 0 & 0 & 0 \\
\hline
\end{tabular}

The results came out as expected: The differences of stiffness, static friction, and dynamic friction were all fully recognized by the users. However, they were highly confused between the stiffness and damping changes. Thus, we concluded that the combination of the two properties defines the hardness of an object, and the damping property has the least influence on the tactile discriminations. 


\subsection{Tactile Texture Recognition Test}

For this test, Haptic Puzzle was used in order to examine tactile texture recognition for the objects in Table 2 . The participants were seven males and three females in their twenties. A target texture was randomly chosen from Table 2 and presented on the screen. They were allowed to probe the target with the haptic device for up to 30 seconds. Then they selected the corresponding texture images among the nine objects in Table 2. The average rate of correct answers is presented in Table 5. In addition, the confusion matrix for this tactile recognition test is shown in Table 6 .

Table 1. Average Correct Answer Rate of Textures

\begin{tabular}{|c|c|c|c|c|c|c|c|c|}
\hline Material & Meat & Jelly & Leather & Glass & Wood & Bone & Orange & Sand \\
\hline $\begin{array}{c}\text { Average } \\
\text { Correct Answer } \\
\text { Rate }\end{array}$ & 100 & 85 & 85 & 75 & & & & 30 \\
\hline
\end{tabular}

Table 2. Confusion Matrix for Tactile Recognition Test

\begin{tabular}{|l|c|c|c|c|c|c|c|c|c|}
\hline Material & Meat & Jelly & Leather & Glass & Mood & Bone & Orange & Rock & Sand \\
\hline Meat & 20 & 0 & 0 & 0 & 0 & 0 & 0 & 0 & 0 \\
\hline Jelly & 0 & 17 & 1 & & 0 & 0 & 2 & 0 & 0 \\
\hline Leather & 1 & 0 & 17 & 0 & 3 & 0 & 0 & 0 & 0 \\
\hline Glass & 0 & 0 & 0 & 15 & 5 & 0 & 0 & 0 & 0 \\
\hline Wood & 0 & 0 & 2 & 1 & 11 & 6 & 0 & 0 & 0 \\
\hline Bone & 0 & 0 & 1 & 0 & 8 & 11 & 0 & 0 & 0 \\
\hline Orange & 0 & 0 & 0 & 0 & 0 & 0 & 9 & 5 & 6 \\
\hline Rock & 0 & 0 & 0 & 0 & 0 & 0 & 6 & 8 & 6 \\
\hline Sand & 0 & 0 & 0 & 0 & 0 & 0 & 7 & 7 & 6 \\
\hline
\end{tabular}

The recognition rates Indicate better results for meat, jelly, glass, leather, bone, wood, orange, sand, and rock indescending order.

The confusion thatyix of the test also presents an interesting piece of information. Wood was often confused with leather or sand. Because we are assessing the four properties of materials, the only difference between wood and sand is the static friction value. Also the differences between wood and leather are the damping and the dynamic friction values. We could thus infer that users cannot differentiate the friction variations very well when the stiffness values of objects are identical.

Examinees were also confused in distinguishing orange, sand, and rock. . The recognition rates of sand and rock were both very low. The static friction value of sand was slightly higher than rock. Such outcome also demonstrates that the users hardly could recognize the fine differences of friction. Another notable result is that wood and bone were confused because their stiffness values were very similar.

An important characteristic of orange was that the static friction and dynamic friction were the same. We could assume that orange was confused with sand or rock because of their similar friction values. However, the recognition rate of orange is relatively high because its stiffness and damping values were particular. The difference of stiffness values between orange (0.5) and sand (1.0) or rock (1.0) was relatively great, and their damping values is 
$(0.1,1.0,1.0$, respectively). This experiment led us to conclude that the stiffness and damping properties are more influential than frictional properties are.

\subsection{Haptic Shape Recognition Test}

There are two main types of solid geometric shapes, "polyhedra" (they must have flat faces), and "non-polyhedra" (if any surface is not flat) as shown in Figure 7. Polyhedra are classified into two main types of cubes and cuboids, platonic solids, prisms, and pyramids. Non-polyhedra are divided into sphere, torus, cylinder, and cone.

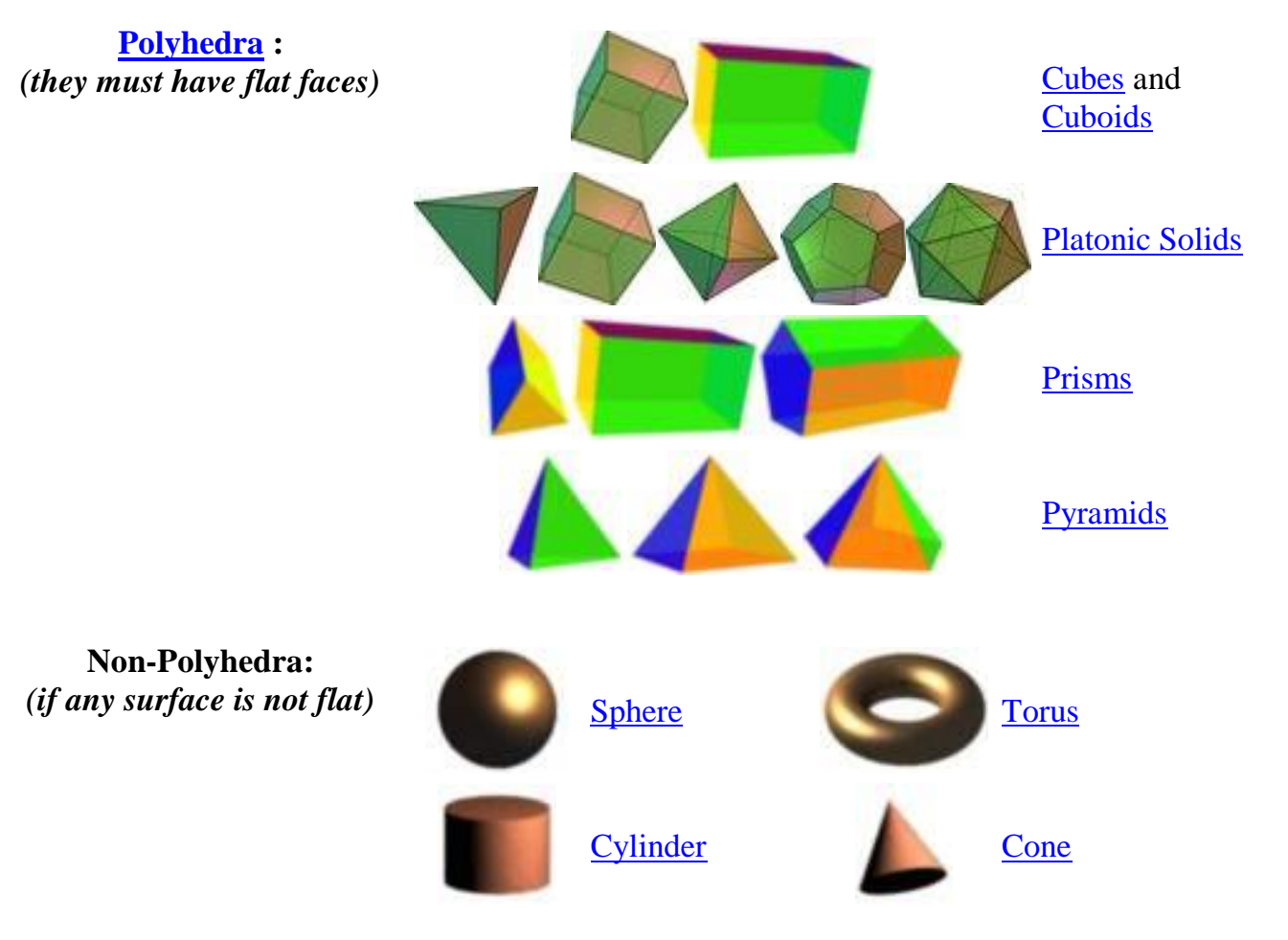

Figure 7. Solid Geometry [18]

This experiment was designed to confirm that our haptic gaming system can help to enhance one's ability to discern different 3D shapes. Our Shape Puzzle was used for this experiment and two non-polyhedra and five polyhedra (platonic solids) were examined. The possible shapes were sphere, torus, tetrahedron (4 triangular faces), hexahedron (6 square faces), octahedron (8 triangle faces), dodecahedron (12 pentagonal faces), and icosahedron (20 triangle faces). Users could probe the assigned 3D object behind the big question mark with a haptic device.

Two cases of this test were experimented on five students. The first case was performed with only spoken information provided about the target objects without any visual information. The second case was performed with the visual knowledge of target shapes given. After a target shape was randomly chosen among the seven figures, the test participants answered what the invisible target shapes were.

Without Visual Information: This experiment was undertaken five times with only the verbal information about the set of 3D shapes (sphere, torus, tetrahedron, hexahedron, octahedron, dodecahedron, and icosahedron). Table 7 illustrates the number of correct 
answers of the shape recognition experiment without any visual information. Figure 8 corresponds to the graphical representation of Table 7. The sphere and the torus received high rates of correct recognition. However, octahedron, decahedron, and icosahedron were not recognized very well. As we repeated the experiment, the rates of correct answers improved except those of dodecahedron and icosahedron. We inferred the reason behind as the shapes being too complex and unfamiliar for the participants.

\section{Table 7. Number of Correct Answers of Haptic Shape Recognition Test without} Visual Information of Target Shapes

\begin{tabular}{|c|c|c|c|c|c|c|c|}
\hline Model & Sphere & Torus & Tetrahedron & Hexahedron & Octahedron & Dodecahedron & Icosahedron \\
\hline $1^{\text {st }}$ trial & 20 & 21 & 10 & 18 & 7 & 3 \\
\hline $2^{\text {nd }}$ trial & 23 & 25 & 11 & 21 & 8 & \\
\hline $3^{\text {th }}$ trial & 25 & 25 & 15 & 23 & & \\
\hline $4^{\text {th }}$ trial & 25 & 25 & 16 & 25 & & \\
\hline $5^{\text {th }}$ trial & 25 & 25 & 19 & 25 & 12 & 4 \\
\hline
\end{tabular}

Figure 8. Graph of Haptic Shape Recognition Test without Visual Information of Target Shapes

With Visual Information: This experiment was also executed five times by providing visual information about the set of $3 \mathrm{D}$ shapes as presented in Figure 9. Table 8 illustrates the number of correct recognitions each figures received with the given visuah information. Figure 10 is the corresponding graphical representation of Table 8. The results indicated that repetitive trials had little influence on improving the recognition rates in this case with visual knowledge.

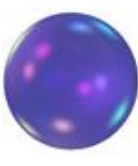

Sphere

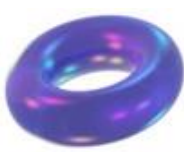

Torus

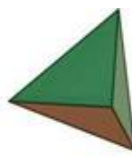

Tetrahedron

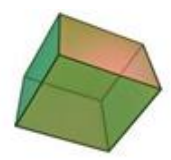

Hexahedron

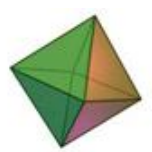

Octahedron

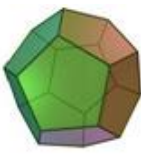

Dodecahedron

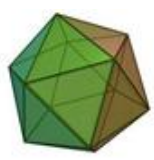

Icosahedron

Figure 9. Target 3D Shapes for Haptic Shape Recognition Test 
Table 8. Number of Correct Answers of Haptic Shape Recognition Test with Visual Information of Target Shapes

\begin{tabular}{|c|c|c|c|c|c|c|c|}
\hline Model & Sphere & Torus & Tetrahedron & Hexahedron & Octahedron & Dodecahedron & Icosahedron \\
\hline $1^{\text {st }}$ trial & 25 & 25 & 23 & 25 & 22 & 16 & 15 \\
\hline $2^{\text {nd }}$ trial & 25 & 25 & 23 & 25 & 23 & 18 & 14 \\
\hline $3^{\text {th }}$ trial & 25 & 25 & 24 & 25 & 23 & 17 & 15 \\
\hline $4^{\text {th }}$ trial & 25 & 25 & 25 & 25 & 25 & 19 & 16 \\
\hline $5^{\text {th }}$ trial & 25 & 25 & 25 & 25 & 25 & 18 & 17 \\
\hline
\end{tabular}

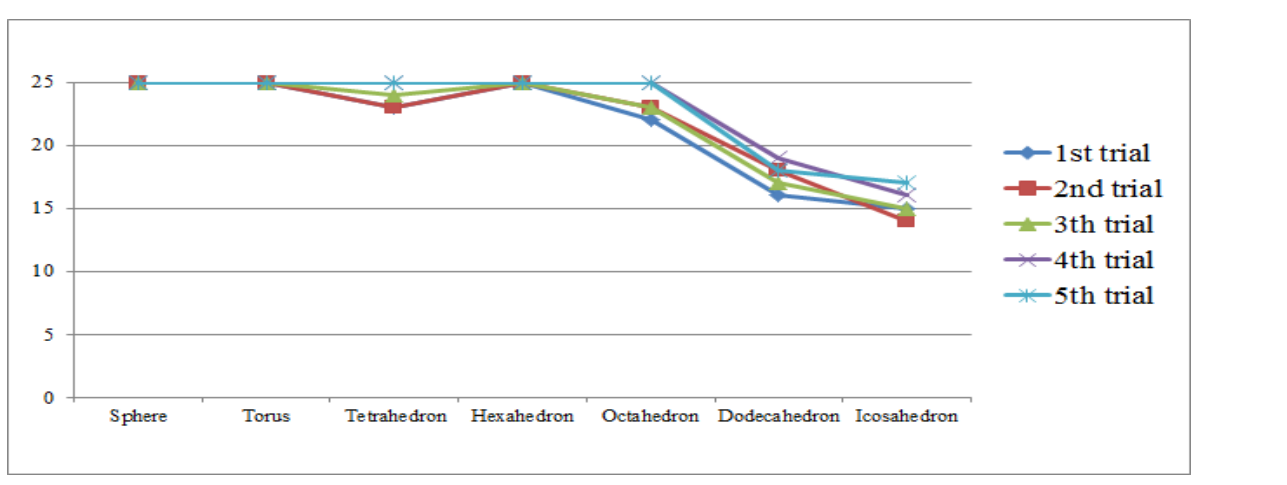

Figure 10. Graph of Haptic Shape Recognition Test with Visual Information of 1 Target Shapes

Figure 11 illustrates the average recognition performance of the two cases: test s with and without visual information. It is notable that the visual knowledge about 3D shapes greatly improved the parficipants' ability to correctly discern the objects.

Figure 11. Graph of Average Recognition Performance with or Without Visual Information

\section{Conclusion}

In this paper, we have proposed a haptic gaming system that can be used to improve one's haptic visual discrimination capability. This system is composed of three components: "Haptic Puzzle" for haptic cognition, "Shape Puzzle" for 3D shape cognition and "Position Puzzle for 3D space cognition.

Numerous experiments were performed to validate our proposal. The first was the system usability test. The Haptic Puzzle received high scores for its entertainingness 
and freshness. The Shape Puzzle earned the highest score in its immersiveness, and the Position Puzzle got similar scores in all the categories. Overall system usability was confirmed by the SUS subjective test, but the users exhibited some difficulties in handling the haptic cursor in 3D space. The unfamiliarity of the haptic device showed that users would require some time for adaptation. Then another test was performed, which was the haptic reality test that measured the recognition of glass, jelly, wood, orange, meat, bone, leather, rock, and sand (the objects are listed in a descending order in terms of how they were successfully recognized by the participants).

From the results of the four haptic properties (stiffness, damping, static friction, and dynamic friction) in the discrimination test, we could infer that the users were least sensitive with damping property, and the damping changes were also highly confused with stiffness changes. Additionally, through the tactile texture recognition test, we found that the stiffness and damping properties are more influental than friction properties for discriminating the textures, even though the friction values defines the different surface characteristics. Then the tactile texture recognition test suggested standard values of the four properties for differept materials by correcting the values of materials that had poor correct answer rates.

Next, our haptic shape recognition test revealed that this gaming system can certainly help to improve one's 3D shape discrimination capability. The experiment with and without the visual information also showed that the visual knowledge of the shapes greatly upgraded the participants' ability to correctly discern the objects.

These experiments allowed us to conclude that our system can help to enhance a person's ability to discern tactile textures and $3 D$ shapes. In addition, the last two recognition experiments that we had conducted are closely related to the HVDT (haptic visual discriminant test) of McCarron-Dial System. It is a standardized and quantitative test kit that is for evaluating cognitive functioning in tactile sensitivity, spatial synthesis, and object integration of partial information into a whole, which can be an effective method to examine the handicapped and the mentally disabled individuals (McCarron \& Dial, 1978) [19]. The HVDT consisted of real tangible components, such as photographic plates representing various objects that had to be identified by the examinees, a folding screen (to obscure objects from the examinee's field of vision), sets of shapes and sizes, textures and configurations, one package of HVDT score forms, and the test manual. Our recognition experiments for tactile textures and 3D shapes demonstrated the feasibility of our system that it can be used for evaluating one's haptic visual discrimination capability and can substitute the expensive HVDT test kit.

Most srgnificantly, our system presented the possibility of the development that can contribute to integrate the vision and haptic sensing of children as well as the physically or mentally disabled. Our final experiment demonstrated that the visuo-haptic integrated recognition was more advantageous than single haptic recognition. Recent advancements in computational neuroscience and brain imaging research have added new insights into the underlying mechanisms and identified possible brain regions involved in visuo-haptic integration [20]. Yet, there still exists a gap between the results from the computational studies and the brain imaging studies. Our experiment can be considered as an attempt to close this gap.

In the future, succeeding researches can possibly focus on the haptic visual discrimination test using our proposed system and incorporating a better understanding of the visuo-haptic integration process. The need for improvement in system usability 
and haptic reality of our system still remains, along with some possible changes of our target 3D geometric shapes into more general ones.

\section{Acknowledgements}

This work was supported by Incheon National University Research Grant in 2011.

\section{References}

[1] J. O. Kim, M. Kim and K. H. Yoo, "Real-Time Hand Gesture-Based Interaction with Objects in 3D Virtual Environments", International Journal of Multimedia and Ubiquitous Engineering, vol. 8, no. 6.(2013)\% pp. 339-348.

[2] S. Andrews, J. Mora, J. Lang and W. S. Lee, "HaptiCast: A physically-based 3d game with haptic feedback", Proceedings of The International Conference on the Future of Game Design and Technology, (2006).

[3] W. Mark, S. Randolph, M. Finch, J. V. Verth and R. M. Taylor, "Adding force feedback to graphics systems: Issues and solutions”, Proceedings of SIGGRAPH 96 Conference, (1996) August, pp. 447-452.

[4] J. K. Salisbury and M. A. Srinivasan, "Phantom-based haptic interaction with virtual objects", IEEE Computer Graphics and Applications, vol. 17, no. 5 (1997) pp.6-19.'

[5] J. H. Crosbie, S. Lennon, M. D. MC McGoldrick, J. Mc Neill, J. W. Burke and S. M. McDonough, "Virtual reality in the rehabilitation of the upper limb after hemiplegic stroke:a andomised pilot study", Proceedings of ICDVRAT, (2008), pp. 229-235.

[6] Y. Takahashi, "Haptic Device System for Upper Limb and Cognitive Rehabilitation - Application for Development Disorder Children", Haptics Rendering and Appheations, Edited Abdulmotaleb El Saddik, InTech (2012), pp. 151-164.

[7] J. David, R. Boian, S. A. Merians, M. Tremaine, G. C. Büdea \$. V. Adamovich, M. Recce and H. Poizner, "Virtual Reality-Enhanced Stroke Rehabilitation", IEEE/Transactions of Neural Systems and Rehabilitation Engineering, vol. 9, no. 3, (2001) September.

[8] V. G. Cobb and P. M. Sharkey, "ADecade of Reselarch and Development in Disability", Virtual Reality and Associated Technologies: Review of ICDVRAT 1996-2006, The International Journal of Virtual Reality, vol. 6, no. 2, (2007), pp. 51-68.

[9] R. Kizony, P. L. Weiss, Neiss, M. Shahar and D. Rand, "TheraGame - a Home Based VR Rehabilitation System", Proceeding of ICDVRAT, (2006), pp. 209-214.

[10] D. Chung, "Cognitive Development Evaluation of Haptic Puzzle Game Using a Haptic Pen. Korea Game Society", vol 9 , no 4,(2009), pp. 45,56)

[11] J. Brooke, "SUS- A quick and dirty usability scale", (1986), pp.189-194.

[12] S. Andrews and J. Lang, Haptic Texturing based on Real-World Samples", IEEE International Workshop on Haptic Audio Visual Environments and thiere Applicaions, (2007) October 12-14, pp. 142-147.

[13] E. Ruffaldi, D. Morris, T.Edmunds, F. Barbagli and D. Pai, "Standardized Evaluation of Haptic Rendering Systems", Proceeding of the Symposium on Haptic Interfaces for Virtual Environment and Teleoperator Systems, (2006) March, pp. 225-232.

[14] M. Mihelj and J. Podobnik, "Haptics for Virtual Reality and Teleoperation”, Springer, (2012).

[15] SensAble Techrologies, PHANTOM Omni Haptic Device, http://www.dentsable.com/haptic-phantomomni.htrn (Retieved June 20, 2014).

[16] OpenGL The Industry's Foundation for High Performance Graphics. http://www.opengl.org (Retieved June $202014)$.

[17] FMOD, FMOD Studio. http://fmod.org (Retieved June 20, 2014).

[18] Solid Geometry, Polyhedra and Non-Polyhedra. http://www.mathsisfun.com/geometry (Retieved June 20, 2014).

[19] L. McCarron and Paul W. Horn, "Haptic visual discrimination and intelligence", Journal of Clinical Psychology, vol. 35, no. 1, (1979) January, pp. 117-120.

[20] J. Aman, C. H. Lu and J. Konczak, "The Integration of Vision and Haptic Sensing: A Computational \& Neural Perspective", Cognitive Critique, vol. 2, (2010) May, pp.75-98. 


\section{Authors}

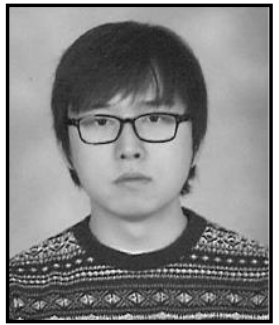

Sung Min Kim, he is a M.S. student at the Department of Computer Science and Engineering, Incheon National University, South Korea. He received his B.S. degree in Computer Science and Engineering from Incheon National University. His research interest includes virtual reality, haptic rendering, 3D Simulations, etc.

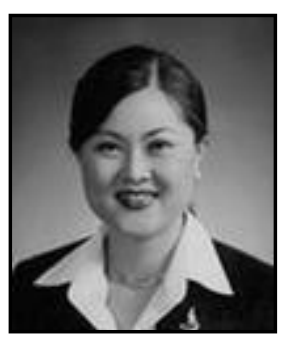

Mee Young Sung, she is a professor at the Department of Computer Science and Engineering, Incheon National University, South Korea. She received her B.S. degree from Seoul National University, South Korea and her M.S. And Ph.D. degrees in Computer Engineering from INSA de Lyon, Erance. Her research interests are haptic virtual reality, haptic audio visual system, telehaptics, multimedia, etc.

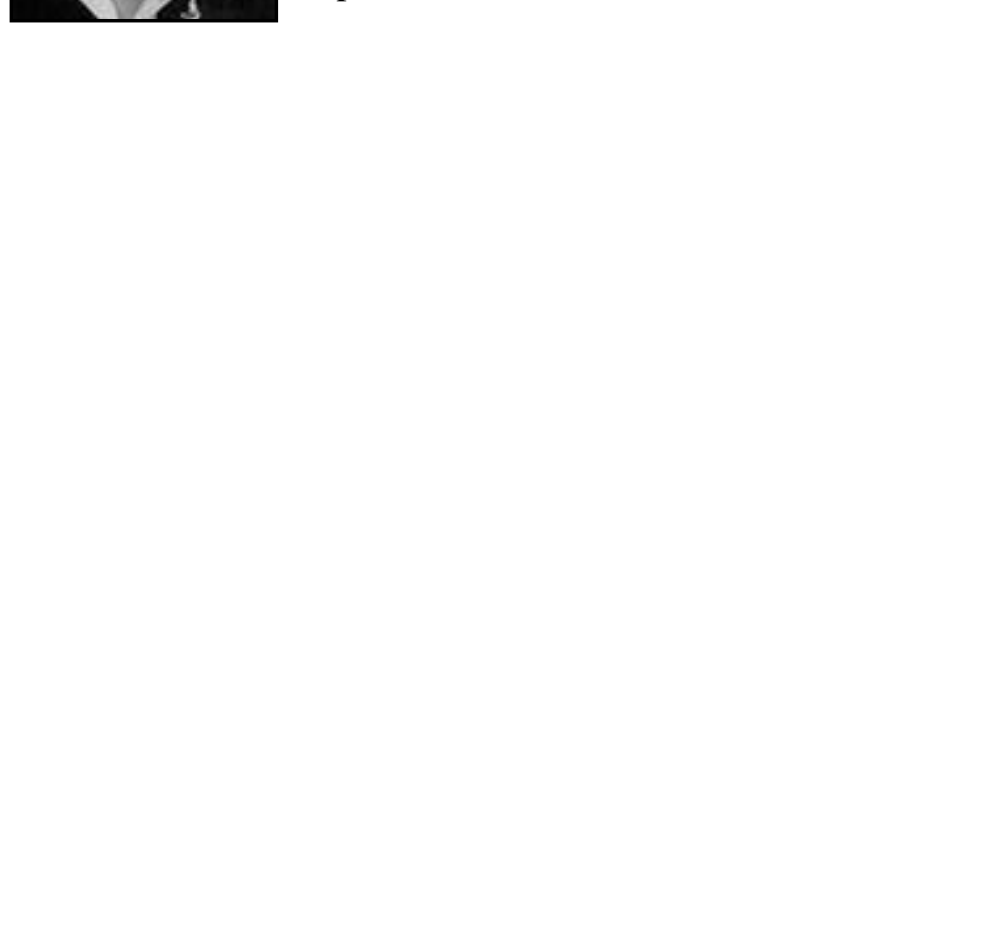


International Journal of Multimedia and Ubiquitous Engineering Vol.9, No.9 (2014)

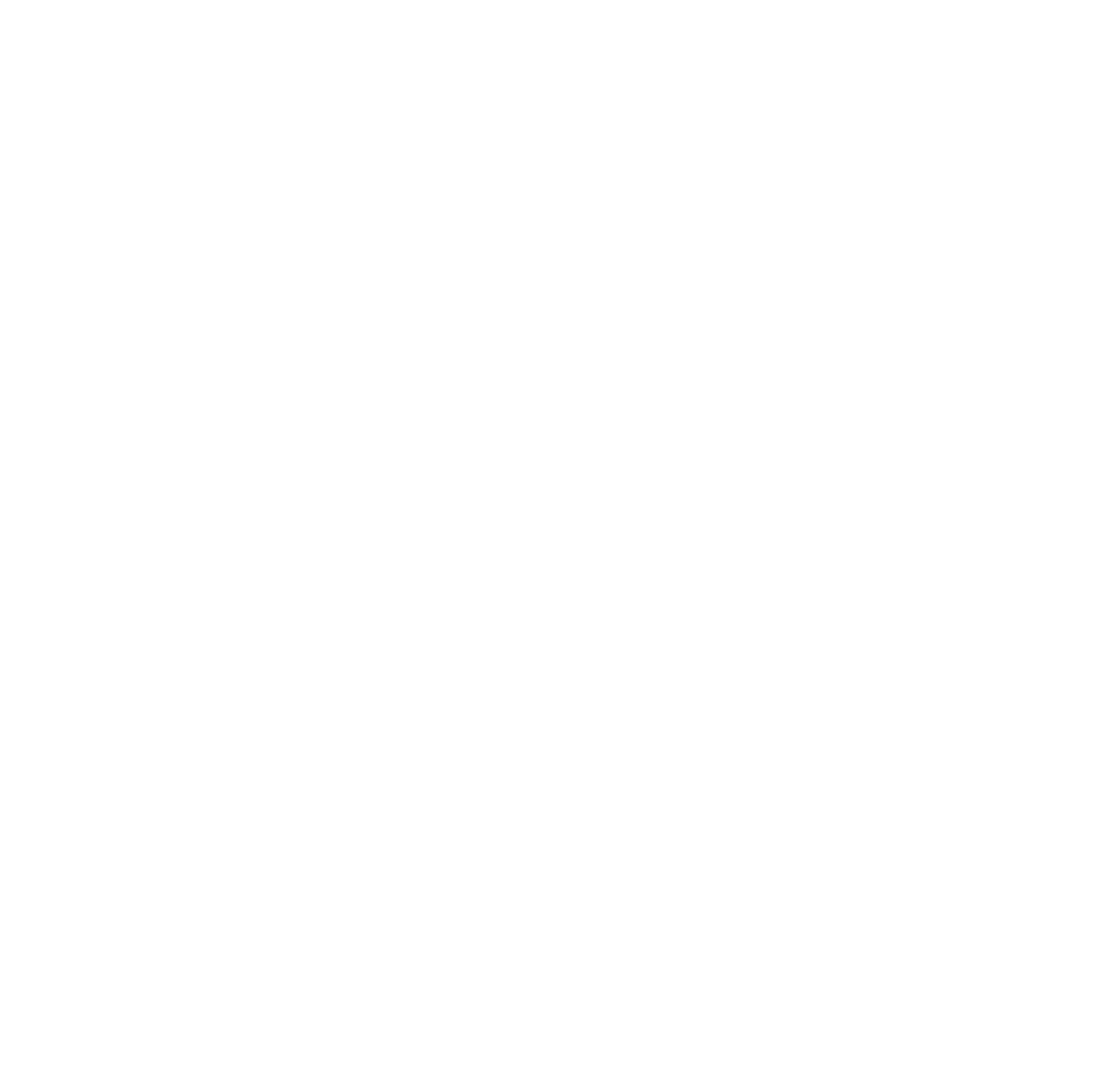

